\title{
Retention of internal experiences in juvenile and adult rats
}

\author{
STEPHEN B. KLEIN, PETER J. MIKULKA, GARY C. DOMATO, and CAROL HALLSTEAD \\ Old Dominion University, Norfolk, Virginia 23508
}

\begin{abstract}
An equal number of adult and juvenile female Sprague-Dawley rats $(n=14)$ received one of four treatments during Phase 1 of the study. Phase 1 consisted of four sucrose-saline pairings (S), four illness experiences (I), four sucrose-illness pairings (SI), or four saline injections (C). After the last Phase 1 treatment, all subjects received a 30-min exposure to sucrose and water followed by a $\mathrm{LiCl}$ injection during the second phase of the experiment. Phase 2 began either 1 or 28 days following the last injection. Each subject continued to receive sucrose-illness experience until a suppression criterion was reached or for a maximum of 4 test days. Adult and juvenile animals that had received sucrose-illness pairings in Phase 1 and tested 1 or 28 days later drank significantly less sucrose than their appropriate control animals. The I treatment adult and juvenile rats at the 1-day retention interval developed the taste aversion more slowly than control animals. Furthermore, the adult, but not the juvenile, rats given sucrose exposures prior to conditioning drank more sucrose than their control rats on Day 2 of training at both intervals. At the 28-day retention test, suppression of sucrose intake was equivalent in adult and juvenile rats in I and $\mathrm{C}$ animals. These results show that young and adult animals can recall prior taste aversion experiences over a 28-day interval but apparently do not remember illness-alone experiences 28 days later.
\end{abstract}

Recent research has focused on the retention of information by young and adult animals over short (i.e., 1 day) and long retention intervals (i.e., 28 days; see Campbell \& Spear, 1972, for complete review of the literature). While recall is equivalent in young (25-30 days old) and adult (approximately 100 days old) rats over a 1-day interval, young animals do not remember prior experiences as well as adult animals over a 28-day retention interval when compared to respective control groups.

Most of the studies investigating retention as a function of age have utilized aversive shock experiences. While adult rats retain such experiences a month later, young rats show poor retention at this interval when compared to their appropriate controls. The present research was designed to explore the retention of another aversive event, illness, by adult and young animals. Taste aversions are rapidly acquired and, once established, tend to be very resistant to extinction.

One explanation of forgetting by young animals over long intervals centers around the concept of interference. Exposure to other environmental cues both prior to and after fear conditioning may be the source of their failure to remember an earlier aversive experience (Campbell \& Spear, 1972). The uniqueness of taste experiences and the relative $a b$ sence of other tastes has been thought to be responsible for acquisition of a taste aversion when illness occurs several hours after taste experience (Revusky, 1971). Galef and Sherry (1973) have reported that weanlings poisoned at the age of 21 days showed an aversion when tested $24 \mathrm{~h}$ later. Since, in this situation, interfering taste experience before and following taste-illness pairing is minimal, it is possible that young animals may be able to recall such taste aversions over a 28-day interval. A primary aim of this study is to determine if young animals can retain a taste aversion over a 28-day retention interval.

Further, Galef and Sherry (1973) found that a 3-day exposure of pups to milk from a mother whose diet was different from the diet of their own mother resulted in a slight increase in preference for the "new" diet when tested 24 h later. Capretta and Rawls (1974) found that pups reared on garlic milk showed a strong preference for this flavor when tested 1 month later. The present study attempted to investigate the retention of a nonpoisoned taste experience by young and adult rats and its effect on the subsequent attempts to establish an aversion to that taste. Based on prior research (Fenwick, Mikulka, \& Klein, 1975), the retention of the prior sucrose experience should interfere with the subsequent establishment of taste aversion.

Finally, a number of studies have demonstrated that preexposure to illness without a contingent taste cue markedly attenuates subsequent attempts to develop taste aversions (Braveman, 1975; Cannon, Berman, Baker, \& Atkinson, 1975; Elkins, 1974; Riley, Jacobs, \& LoLordo, 1976). The rats may have learned during preexposure that illness can occur without prior taste, and thus the no tasteillness experience interferes with a subsequent tasteillness association. The proposed research will in- 
vestigate whether young and adult animals can retain the memory of an illness-alone experience over 1and 28-day intervals.

\section{METHOD}

\section{Subjects}

The subjects were 112 experimentally naive female rats purchased from Sprague-Dawley, Inc. (Madison, Wisconsin). At the onset of the study, half of the rats were 23 days old and the other half were 65 days old. All subjects were individually caged and maintained on an ad-lib food schedule.

\section{Procedure}

Upon arrival in the laboratory, all animals were given 1 day of ad-lib access to water and were then placed on a 23.5-h waterdeprivation schedule for 3 consecutive days. Beginning on the 5th day, the experimental manipulations began. Fourteen juveniles and 14 adults were assigned to each of the four experimental conditions: sucrose-illness pairings (SI), sucrose-saline pairings (S), illness-alone experiences (I), and controls receiving water followed by saline injections (C). Animals in the SI condition were given access to both a $10 \%$ sucrose solution and water for $15 \mathrm{~min}$ followed by $15 \mathrm{~min}$ of access to water. After this, each rat received an intraperitoneal injection of a $.15 \mathrm{~m} \mathrm{LiCl}$ in an amount equal to $2 \%$ of their body weights. This free choice training procedure is identical to one employed in an earlier study to develop a taste aversion in juvenile and adult rats (Klein, Domato, Hallstead, Stephens, \& Mikulka, 1975). All SI animals continued to receive sucrose and water access every other day for 4 days (conditioning Days 1, 3, 5, and 7). On alternate days, all subjects were allowed $30 \mathrm{~min}$ access to water (Days 2, 4, 6, and 8). Lithium chloride injections followed sucrose/water access if more than $1 \mathrm{~g}$ of sucrose solution was consumed on any given day.

Animals in the $\mathrm{S}$ groups received a similar treatment, with the following exception: the animals were given $30 \mathrm{~min}$ of access to the sucrose and water solutions followed by an isotonic saline injection. Similarly, the I subjects received $30 \mathrm{~min}$ of water access followed by $\mathrm{LiCl}$ injections every other day for 4 days. Lastly, the $\mathrm{C}$ groups received $30 \mathrm{~min}$ access to water followed by an isotonic saline injection. On alternate days, all groups received 30 min access to water.

After receiving the appropriate experimental or control treatment, an equal number $(n=7)$ of juvenile and adult rats from each treatment group were tested for retention of their prior experiences either 1 or 28 days following the last treatment day (Day 7). All animals were treated identically during testing. During testing, sucrose and water were available for $30 \mathrm{~min}$ followed by an injection of lithium chloride. All rats continued to receive this sucrose-illness treatment until sucrose consumption dropped to $1 \mathrm{~g}$ or less on any given day or after a maximum of 4 conditioning days. Water was available for $30 \mathrm{~min}$ on days following illness. Animals given the 28-day retention test were placed on ad-lib water following the last treatment day, with a 23.5-h water-deprivation schedule introduced 3 days prior to testing. Mean sucrose intakes on the first day of testing were calculated for adult and juvenile control groups at each retention interval. The percentage of the Day 1 control intake was then calculated for each rat over the 4 days of testings, using the appropriate control group for the given age and retention interval (i.e., the control group for the S-I adult rats tested at the 1-day interval was the adult control group tested on the 1-day test). The percentage of the first test day control intakes was then used for statistical analysis.

\section{RESULTS}

Individual 2 (Age) by 2 (Retention Interval) by 4 (Treatment) factorial analyses of variance were

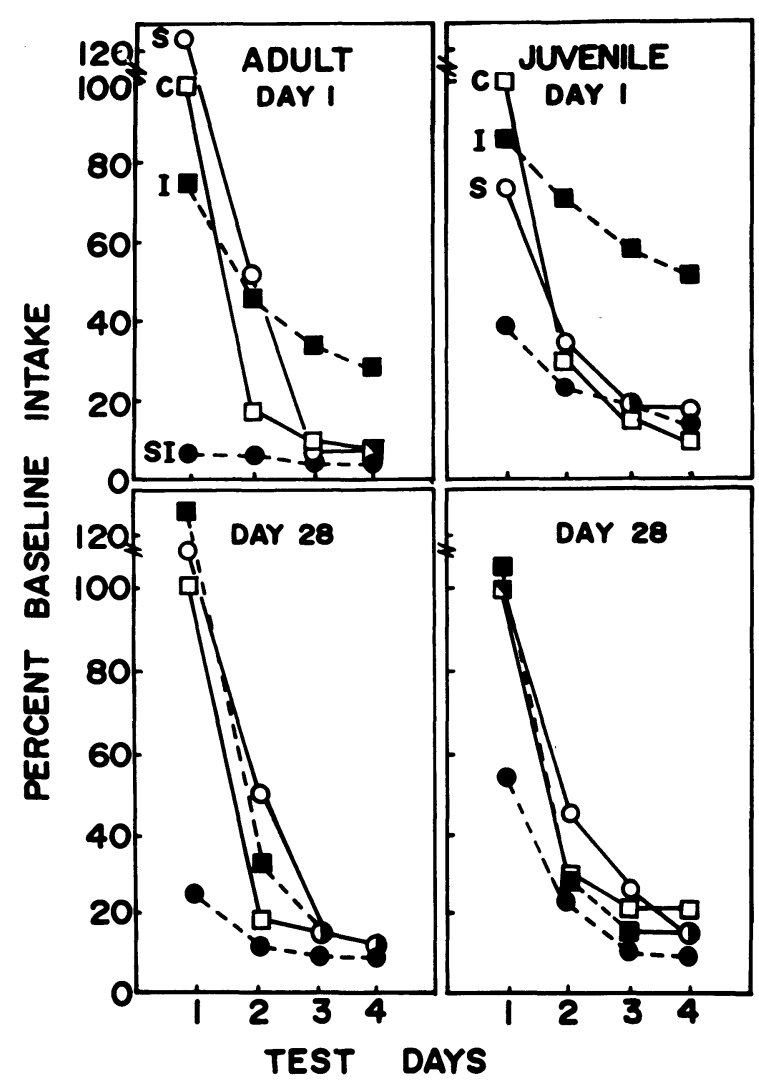

Figure 1. Mean percent baseline intake during four sucrose$\mathrm{LiCl}$ pairings as a function of age, pretesting treatment, and retention interval.

performed on each of the 4 days of retention testing (see Figure 1). Analysis of the Day 1 percentage data yielded significant main effects for Retention Interval $[\mathrm{F}(1,94)=4.41, \mathrm{p}<.05]$ and Treatment $[\mathrm{F}(3,94)$ $=24.9, \mathrm{p}<.005]$. The latter term reflects the significantly lower intakes of the SI subjects compared to the other three treatments, which were not different from each other (Duncan ranges). Also, a significant Age by Treatment interaction was found $[F(3,94)=$ $2.9, \mathrm{p}<.05$ ], which reflects the fact that the adult SI animals had the lowest intake while the adult $\mathrm{S}$ groups had the highest intakes, with the control and juvenile SI and S groups intermediate. Duncan range tests revealed that the $S, I$, and $C$ groups were not different at any age or retention interval. However, they all drank significantly more sucrose $(p<.01)$ than did the SI animals.

All SI groups had equivalent percentage intakes. The apparent high mean intake for the juvenile SI rats on the 28-day tests seen in Figure 1 was due to the intake of one animal whose intake was $167 \%$ of baseline control consumption. The mean percentage intake in SI juvenile rats without the subject was $34 \%$ (numerically equivalent to the intake of the juvenile SI rats at the 1-day test). In addition, the tendency for higher percentage intakes in the 
juvenile when compared with adult rats on the 1-day test was due to the fact that a few of the juvenile SI animals had not reached the $1 \mathrm{~g}$ or less suppression criterion by the end of training (after four SI pairings).

The factorial analysis of Day 2 yielded significant effects for Retention Interval $[F(1,94)=4.8, p<.05]$, for Treatment $[F(3,94)=17.6, p<.0005]$, and for the Treatment by Retention Interval interaction $[F(3,94)=5.1, p<.01]$. Range tests indicated that the SI and $C$ groups were not different from each other, but had lower intakes than was found in all the other groups. Also, the I subjects tested at the 1-day interval had higher intakes than the I 28-day test subjects, while the $S$ groups were not different as a function of the retention interval and were intermediate between both I retention groups.

The Age by Treatment interaction was significant $[F(3,94)=22.5, p<.0005]$, which primarily reflects the pattern of the $S$ and I groups as a function of age. Of these latter groups, the juvenile $S$ subjects had lower intakes than the juvenile I subjects, while this pattern is reversed in the adult subjects, with the adult $\mathrm{S}$ subjects showing the highest percentage intakes. There was a significantly higher percentage baseline intake in the sucrose adult subjects at both retention intervals, but the juvenile sucrose animals displayed equivalent intakes to that found in the control animals at both the 1- and 28-day retention intervals. Intake of sucrose in the I groups was above that of the control rats on the 1-day test in juvenile and adult rats, but equal to that of the control rats at both ages on the 28-day test.

On Day 3, the factorial analysis yielded significant main effects for Age [F(1,94) = 18.1, $\mathrm{p}<.0005]$, Treatment $[F(3,94)=16.5, p<.0005]$, and Retention Interval $[F(3,94)=10.7, p<.0005]$. The Age by Treatment interaction $[\mathrm{F}(3,94)=22.5, \mathrm{p}<$ .0005 ] was also significant. The Duncan's range tests indicated that the I subjects given illness-alone experience drank more sucrose than all other groups on the 1-day test, while there were no differences in percent intakes on the 28-day test. The pattern of intakes for the Day 4 test mirrored that of Day 3.

Examination of total fluid intakes (sucrose and water) revealed that total intakes were equivalent for all animals within each age and retention interval. Thus, all juvenile rats on the 1-day test or on the 28-day test drank equivalent amounts of fluid (sucrose and water). Similarly, there were no significant differences in total intake among the adult groups at either the 1- or the 28-day tests. Additionally, while adult rats had equivalent total intakes on the 1 - and 28-day tests, total fluid consumption was greater for the juvenile rats on the 28-day test than those on the 1-day test. It was apparent that as sucrose intake declined, water consumption increased, thereby maintaining a relatively constant fluid intake. These results indicate that observed differences in sucrose consumption were not due to a general malaise or a nonspecific fluid reduction but specifically due to aversion to sucrose.

\section{DISCUSSION}

Retention of aversive shock experience after long intervals (i.e., 1 month) is poorer in young animals than in adult rats. Forgetting by young animals appears to be a robust phenomenon. Explanations of this phenomenon center around changes in physiological processing occurring and during the retention interval and greater sources of interference for young animals (Campbell \& Spear, 1972).

The results of this study are inconsistent with previous developmental retention research. Young animals were able to recall an acquired taste aversion as well as were adult rats over a 28-day retention interval. Sucrose intake was significantly lower in the juvenile and the adult rats in the SI groups at both the 1- and 28-day tests than was observed in their appropriate control animals. Unlike the retention of fear stimuli, young rats can remember an aversion to a taste over a 28 -day interval. Given the importance of diet selection to species survival, longterm retention of aversions by young animals would be an adaptive characteristic. Other research has shown that young animals can acquire aversions through interactions with their mother (Bronstein, Levine, \& Marcus, 1975). Some mechanism for retention of these experiences may develop at an early stage of ontogenetic development. It may be that physiological systems processing taste experiences develop prior to the development of processes responsible for fear memories. The guinea pig is physiologically mature at birth, and juvenile guinea pigs show no forgetting of prior fear experiences (Campbell, Misanin, White, \& Lytle, 1974). The gustatory and olfactory system in the rat may develop early, and retention by juvenile rats of taste aversions may not be adversely influenced by physiological and neural brain changes.

The absence of interference with long-delay CS-UCS intervals has been attributed by Revusky (1971) to the fact that typically, following consumption of a taste, no other tastes are experienced to interfere with the development of the taste aversion. Revusky has found that vinegar presented during the CS-UCS interval attenuates the aversion to sucrose. The absence of other aversions during the 28-day retention interval may also be responsible for long-term retention of a sucrose aversion by juvenile rats. Since interference is thought to be one source of forgetting of fears by juvenile rats over a 28-day interval, retention of taste aversions may be the result of an absence of interfering agents.

Prior experience with illness without any associ- 
ated taste produced a marked attenuation of sucrose aversion in both juvenile and adult rats on the 1-day test. This clearly indicates that both juvenile and adult animals (I groups) can recall prior unpaired illness experience 1 day later. Adult rats in the S group drank more sucrose after one sucrose-illness pairings than did their control rats, which indicates that prior sucrose exposure (familiarization) was recalled by adult rats on the 1-day test. However, when juveniles were compared to their controls, there was no evidence of such a familiarization effect at the 1-day test. Therefore, retention of the prior sucrose experience interfered with the establishment of an aversion in adult, but not in juvenile, rats.

The inability of juvenile rats to recall prior sucrose experience on the 1-day test is surprising, since Capretta and Rawls (1974) observed that juvenile rats retain a preference for mother's milk over a 33-day interval. Capretta and Rawls (1974) used a minimum of 5 days of postweaning sucrose experience with a maximum of 24 days of sucrose exposure (which includes the 19 days of nursing). This extended exposure, compared to the present study, may account for the discrepancy in observed retention of sucrose-only exposures in the $S$ juvenile rats on the 1-day test. Whether juvenile rats are capable of remembering prior sucrose experiences 28 days later is an empirical question which must be preceded by the demonstration of the retention of sucrose experience over the 1-day interval. The failure of juvenile rats to show a familiarization effect on the 1-day test may be due to the degree of original learning, since this variable has been shown to play an important role in retention of aversive shock experiences (Spear, 1973). A higher degree of learning (more sucrose exposure) might result in the retention of prior sucrose experiences over at least a 1-day interval in juvenile rats. Future research should determine whether increased amounts of sucrose exposure is critical for the retention of a sucrose experience at the 1-day test by juvenile rats.

Although both the juvenile and adult rats appear to remember a taste aversion over a 28-day interval, neither group showed any evidence for the retention of prior unpaired illness experience at this interval. The rate of acquisition of a sucrose aversion on a 28-day test was equivalent for both ages in the unpaired illness condition when compared to their respective control group animals. In addition, a sucrose familiarization effect was observed in adult rats at both retention intervals, while intake of sucrose was equal in $S$ and $C$ juvenile rats at 1 - and 28-day tests. Therefore, with few exceptions, the major results of this study indicated that retention of experiences in taste aversion situations is not related to age. Both adult and-juvenile animals appear to be capable of remembering a prior aversion to sucrose 28 days later, while retention of prior unpaired illness experience was not recalled over a 28-day interval in either adult or juvenile rats.

\section{REFERENCES}

Braveman, N. S. Formation of taste aversions in rats following prior exposure to sickness. Learning and Motivation, 1975, 6, 512-534.

Bronstein, P. M., Levine, M. J., \& Marcus, M. A rat's first bite: The nongenetic, cross generational transfer of information. Journal of Comparative and Physiological Psychology, 1975, 89, 295-298.

Campbell, B. A., Misanin, J. R., White, B. C., \& Lytle, L. D. Species differences in the ontogeny of memory: Indirect support for neural maturation as a determinant of forgetting. Journal of Comparative and Physiological Psychology, 1974, 87, 193-202.

Campbell, B. H., \& Spear, N. E. Ontogeny of memory. Psychological Review, 1972, 79, 215-236.

Cannon, D., Berman, R., Baker, T., \& Atrinson, C. Effect of preconditioning unconditioned stimulus experience on learned taste aversions. Journal of Experimental Psychology: Animal Behavior Processes, 1975, 104, 270-284.

CAPRetta, P., \& RAwls, L. Establishment of a flavor preference in rats: Importance of nursing and weaning experience. Journal of Comparative and Physiological Psychology, 1974, 86, 670-673.

ElkINS, R. L. Bait shyness acquisition and resistance to extinction as a function of US exposure prior to conditioning. Physiological Psychology, 1974, 2, 341-343.

Fenwick, S., Mikulka, P. J., \& Klein, S. B. The effect of different levels of pre-exposure to sucrose on the acquisition and extinction of a conditioned aversion. Behavioral Biology, 1975, 14, 231-235.

GAlef, B., JR., \& Sherry, D. Mother's milk: A medium of transmission of cues reflecting the flavor of mother's diet. Journal of Comparative and Physiological Psychology, 1973, 78, 213-219.

Klein, S. B., Domato, G. C., Hallstead, C., Stephens, I., \& Mrkulka, P. J. Acquisition of a conditioned aversion as a function of age and measurement technique. Physiological Psychology, 1975, 3, 379-384.

REVUSKY, S. H. The role of interference in association over a delay. In W. K. Honig \& P. H. R. James (Eds.), Animal memory. New York: Academic Press, 1971. Pp. 155-213.

Riley, A. L., Jacobs, W. J., \& Lolordo, V. M. Drug exposure and the acquisition and retention of a taste aversion. Journal of Comparative and Physiological Psychology, 1976, 90, 799-807.

SPEAR, N. E. Retrieval of memory in animals. Psychological Review, 1973, 80, 168-194.

(Received for publication February 3, 1976; revision accepted November $8,1976$. 